\title{
Model Design of Piezoelectric Micromachined Modal Gyroscope
}

\author{
Xiaojun Hu, Xiaosheng Wu, Zheng Wang, Wenyuan Chen, and Weiping Zhang \\ National Key Laboratory of Micro/Nano Fabrication Technology, Research Institute of Micro/Nano Science and Technology, \\ Shanghai Jiao Tong University, Shanghai 200240, China
}

Correspondence should be addressed to Xiaosheng Wu, xswu@sjtu.edu.cn

Received 30 May 2011; Revised 30 August 2011; Accepted 30 August 2011

Academic Editor: Andrea Cusano

Copyright (C) 2011 Xiaojun Hu et al. This is an open access article distributed under the Creative Commons Attribution License, which permits unrestricted use, distribution, and reproduction in any medium, provided the original work is properly cited.

This paper reports a novel kind of solid-state microgyroscope, which is called piezoelectric micromachined modal gyroscope (PMMG). PMMG has large stiffness and robust resistance to shake and strike because there is no evident mass-spring component in its structure. This work focused on quantitative optimization of the gyroscope, which is still blank for such gyroscope. The modal analysis by the finite element method (FEM) was firstly conducted. A set of quantitative indicators were developed to optimize the operation mode. By FEM, the harmonic analysis was conducted to find the way to efficiently actuate the operational mode needed. The optimal configuration of driving electrodes was obtained. At last, the Coriolis analysis was conducted to show the relation between angular velocity and differential output voltage by the Coriolis force under working condition. The results obtained in this paper provide theoretical basis for realizing this novel kind of micromachined gyroscope.

\section{Introduction}

Different from the micromachined vibratory gyroscope, which has the structure of suspending springs and proof masses, some kinds of solid-state gyroscopes have no part which moves as a whole. According to their working principle, these kinds of gyroscopes can be classified into two categories: one that has no vibration unit at all such as optical gyroscopes [1] and atom gyroscope [2,3], the other that has only local vibration such as HRG (Hemispherical Resonant Gyro) [4] and surface acoustic wave gyroscope [5, 6]. Because there is no moving part and suspending structure in a solidstate gyroscope, it is robust and has higher resistance to shock and shake. As the development of MEMS technology, MEMS microgyroscopes attracted increasing attention due to their unique advantage (low price, small size, low energy consumption, and easy integration) and it sees more and more research on miniaturization of solid-state gyroscopes. Solid-state microgyroscopes combine the merits of both solid-state gyroscopes and micro-MEMS gyroscopes. At present, there are four major solid-state microgyroscopes: micro-HRG (Hemispherical Resonator Gyro), micro-optical gyroscopes $[7,8]$, microsurface acoustic wave gyroscopes $[6$, 9], and piezoelectric solid-state microgyroscopes researched in the paper.
Piezoelectric gyroscopes make use of two vibration modes of a vibratory piezoelectric body in which material particles move in two perpendicular directions, respectively. When a piezoelectric gyroscope is excited into vibration in one of the two modes (the primary mode) by an applied alternating voltage and attached to a rotating body, Coriolis force will excite the other mode (the secondary mode) through which the rotation rate of the body can be detected. These piezoelectric gyroscopes include flexural vibrations in two perpendicular directions of beams and tuning forks [10-13], thickness-shear vibrations in two perpendicular directions of plates $[14,15]$, radial and torsion vibrations of circular cylindrical shells [16], and degenerate modes of circular disks, shells, and rings [17-19].

In January of 2006, Japanese researchers Maenaka et al. proposed a novel piezoelectric solid-state microgyroscope [20], which is named the piezoelectric micromachined modal gyroscope (PMMG) in this paper. It was found that in a higher-order resonance mode, the movements of the mass elements are almost in one direction and differential on the sides of the prism, and this modal vibration can be used to detect the rotation rate of the body, which will be detailed below. This kind of novel gyroscope has attracted many attention, and large amount of both theoretical and experimental researches have been done [21-23]. However, 


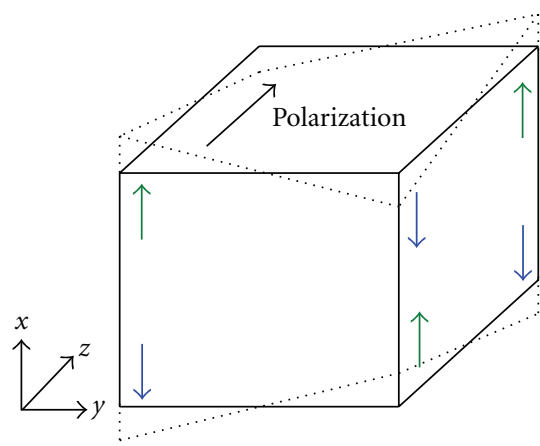

(a)

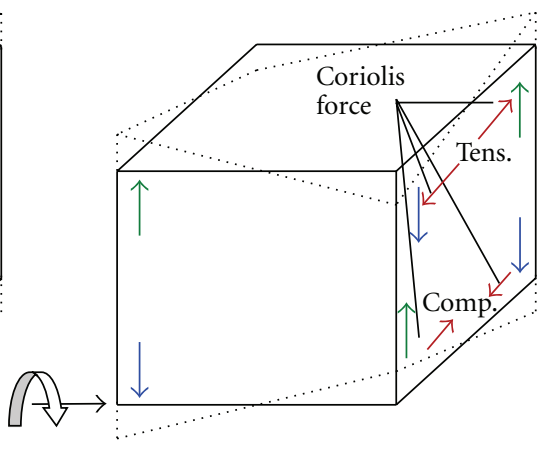

(b)

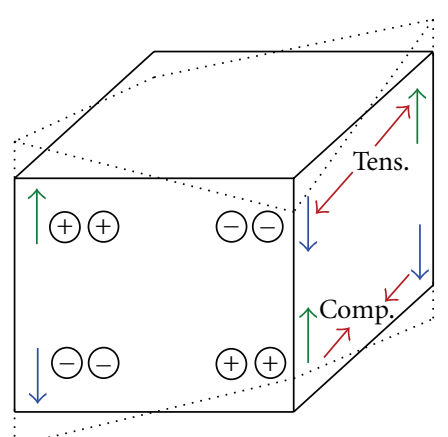

(c)

FIGURE 1: Operation principle of the gyroscope.

these works mainly focused on qualitative researches, and quantitative analysis and optimization of such gyroscope is still blank.

In this paper, quantitative indicators were introduced to evaluate the modes and thus determine the best operational mode. Besides, the same indicators were used to determine the size and configuration of the driving electrodes with which the operational mode can be efficiently actuated. At last, the Coriolis analysis was conducted to show the relation between angular velocity and differential output voltage by the Coriolis force under working condition.

\section{Operation Principle}

The basic operation principle of the device we applied is shown in Figure 1. At first, assume that the rectangular prism is made of PZT (a piezoelectric ceramics made of $\mathrm{PbO}_{2}$, $\mathrm{PbZrO}_{3}$ and $\mathrm{PbTiO}_{3}$ ), and it has polarization along $z$-axis. With excitation of the PZT prism at the operation mode mentioned before, the differential vibration to $x$-axis (in our simulation, we use $y$-axis) in the prism appears as shown in Figure 1(a). When the angular velocity along $y$-axis (in our simulation we use $x$-axis) is applied, the Coriolis force is generated according to movement of the mass elements, resulting in compressive and tensile stresses depending on the position. These stresses differentially generate the piezoelectric voltage at the surface of the device as shown in Figure 1(b). This voltage is proportional to the applied angular rate and is an output signal of the device.

The detail structure of the piezoelectric solid-state microgyroscope is introduced as shown in Figure 2, which was first proposed by Maenaka et al. Piezoelectric ceramic material PZT is selected as the substrate block. On the top and bottom surfaces of the PZT block, a lot of driving electrodes and sensing electrodes are distributed. As shown in the figure, D+ and $\mathrm{D}-$ are the driving electrodes, and the exciting voltages are applied on the $\mathrm{D}+$ and $\mathrm{D}-$ electrodes. $\mathrm{R} 1$ and $\mathrm{R} 2$ are the reference electrodes, which can be used for searching and tracking the working resonance mode. S1, S2, S3, and S4 are the sensing electrodes. When there is no angular input, the voltages of two adjacent electrodes, such as S1 and S2, or S3 and S4, are the same because of the symmetric structure

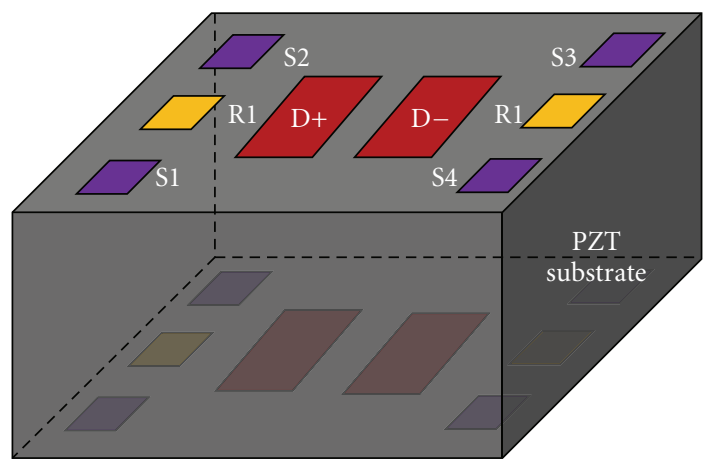

FIGURE 2: Detail structure of the piezoelectric solid-state microgyroscope.

of the piezoelectric block. If the rotation in any direction perpendicular to the modal vibration is input, the voltage of sensing electrodes is changed because of the coupling of the Coriolis effect, and then the voltages of two adjacent electrodes are not the same. Through detecting the voltage difference of two adjacent sensing electrodes, the rotation input can be quantized. On the bottom surface, the sensing and driving electrodes are distributed in the corresponding positions as on the top surface.

\section{Design and Simulation}

3.1. Material Selection. Piezoelectric material is commonly used for electromechanical transducers, and the requirements for the performance of piezoelectric ceramic vary for different regions of applications. As for the solid-state microgyroscope we used, the piezoelectric material serves as the excitation source and sensing element simultaneously, so the piezoelectric material should have larger piezoelectric constant $d_{33}$ and electromechanical coupling constants $k_{33}$ and $k_{15}$. Based on these conditions, we selected PZT-5 during following simulation. The PZT-5 material constants used in this paper are listed as follows: the dielectric constant $\left(\varepsilon_{r}\right): \varepsilon_{11}=1700, \varepsilon_{33}=1470$, material mass density $(\rho)$ : $7500 \mathrm{~kg} / \mathrm{m}^{3}$, the piezoelectric constant $(e): e_{31}=-6.5 \mathrm{C} / \mathrm{m}^{2}$, $e_{33}=23.3 \mathrm{C} / \mathrm{m}^{2}, e_{15}=17.0 \mathrm{C} / \mathrm{m}^{2}$, the elastic constant 
matrix $(c): c_{11}=12.6 \times 10^{10} \mathrm{~N} / \mathrm{m}^{2}, c_{12}=7.95 \times 10^{10} \mathrm{~N} / \mathrm{m}^{2}$, $c_{13}=8.41 \times 10^{10} \mathrm{~N} / \mathrm{m}^{2}, c_{33}=11.7 \times 10^{10} \mathrm{~N} / \mathrm{m}^{2}, c_{44}=$ $2.30 \times 10^{10} \mathrm{~N} / \mathrm{m}^{2}, c_{66}=2.325 \times 10^{10} \mathrm{~N} / \mathrm{m}^{2}$.

3.2. Modal Analysis. In this section, the finite element analysis of the piezoelectric body of the PMMG was first conducted to find the operation mode, and then quantitative indicators were introduced to evaluate these modes and at last the best operation mode and the corresponding size of the device was given.

From the operation principle of the PMMG, it can be concluded that the working resonance mode should have the following characteristics. (1) The movement of points in the piezoelectric block should be almost in one direction, $y$-axis in this paper. (2) The moving direction of points should be perpendicular to the polarization direction of the piezoelectric block. (3) The moving direction of a point on one edge is the same as that of the corresponding point on the diagonal edge and is opposite to that of the corresponding point on the adjacent edge. (4) Moving edges should be in the state of tension or compression. To get this special modal shape, we used ANSYS to perform modal analysis and list corresponding frequencies under which the modal shapes meet these characteristics. The corresponding results are listed at Table 1.

As can be seen from Table 1, 9 results were obtained, and it raises a question that which one is the best. For the ideal working resonance mode, the points vibrate only in $y$-axis direction and there is no vibration in other two orthogonal directions. Actually, that is impossible and we hope the oscillation amplitude is much bigger at $y$-axis direction than at $x$-axis and $z$-axis directions. For that reason, we established a set of quantitative indicators to evaluate various modes and thus determine the best operation mode.

Before we introduce these quantitative indicators, it is necessary to clarify that the device was meshed before simulation was conducted; therefore, it is reasonable to think the device was composed of many small units and nodes.

The quantitative indicators are shown as follows:

$$
\begin{aligned}
& A=\frac{\sum E_{Y i}}{\sum E_{X i}}, \\
& B=\frac{\sum E_{Y i}}{\sum E_{Z i}},
\end{aligned}
$$

where $E_{Y i}, E_{X i}$, and $E_{Z i}$ stand for the kinetic energy of element $i$ at $Y, X$, and $Z$ directions, respectively, $A$ and $B$ are our quantitative indicators and they are the ratio of $E_{Y}$ to $E_{X}$ and $E_{Z}$, respectively. When the value of $A$ and $B$ gets bigger, that means the movements of the mass elements are more obvious at $Y$ direction than at $X$ and $Z$ directions, which leads to higher sensitivity and lower deviation.

To calculate $A$ and $B$, we need to get $E_{Y i}, E_{X i}$, and $E_{Z i}$. We started from calculating displacement of element $i$. Displacement of element $i$ at $X$ direction is shown in (2):

$$
U_{X i}=A_{X i} \sin (w t+\varphi) .
$$

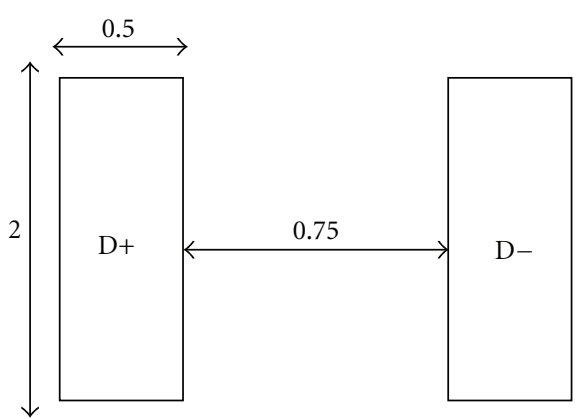

Figure 3: The configuration of the driving electrodes.

Through derivation, we can calculate velocity of element $i$ at $X$ direction:

$$
V_{X i}=A_{X i} w \cos (w t+\varphi) .
$$

Using Kinetic Energy Theory, we got the kinetic energy of element $i$ at $X$ direction:

$$
E_{X i}=\frac{1}{2} m V_{X i}^{2}=\frac{1}{2} m A_{X i}^{2} w^{2} \cos ^{2}(w t+\varphi) .
$$

In the same way, we got $E_{Y i}$ and $E_{Z i}$ :

$$
\begin{aligned}
& E_{Y i}=\frac{1}{2} m V_{Y i}^{2}=\frac{1}{2} m A_{Y i}^{2} w^{2} \cos ^{2}(w t+\varphi), \\
& E_{Z i}=\frac{1}{2} m V_{Z i}^{2}=\frac{1}{2} m A_{Z i}^{2} w^{2} \cos ^{2}(w t+\varphi) .
\end{aligned}
$$

Using (1)-(5), we got $A$ and $B$ as shown below:

$$
\begin{aligned}
& A=\frac{\sum E_{Y i}}{\sum E_{X i}}=\frac{\sum A_{Y i}^{2}}{\sum A_{X i}^{2}}, \\
& B=\frac{\sum E_{Y i}}{\sum E_{Z i}}=\frac{\sum A_{Y i}^{2}}{\sum A_{Z i}^{2}} .
\end{aligned}
$$

$A_{X i}, A_{Y i}$, and $A_{Z i}$ can be got from ANSYS, and they are relative values because modal analysis does not give us absolute values generally.

Table 2 shows the calculating results of quantitative indicators.

In order to get the best performance, we hope the value of $A$ and $B$ as well as $A_{Y}$ reaches its maximum value simultaneously, but in real applications that is impossible and unnecessary. A practical way is to make an overall comparison of these indicators among all the modes listed in Table 2 and choose the relatively biggest one. As can be seen from Table 2 , the device with the size $3 \times 4 \times 4$ has the maximum value of $A$ and $A_{Y}$, and its value of $B$ is smaller than only that of the device with the size $3 \times 6 \times 5$. Besides, the device with the size $3 \times 5 \times 4$ also has relatively big value of $A, B$, and $A_{Y}$. Another factor to consider is that because of symmetry, the device with the size $3 \times 4 \times 4$ will have two mode shapes at the same frequency. As a result, we can determine that the device with the size $3 \times 5 \times 4$ has the best operation mode. 
TABle 1: Particular mode shapes meeting our needs.

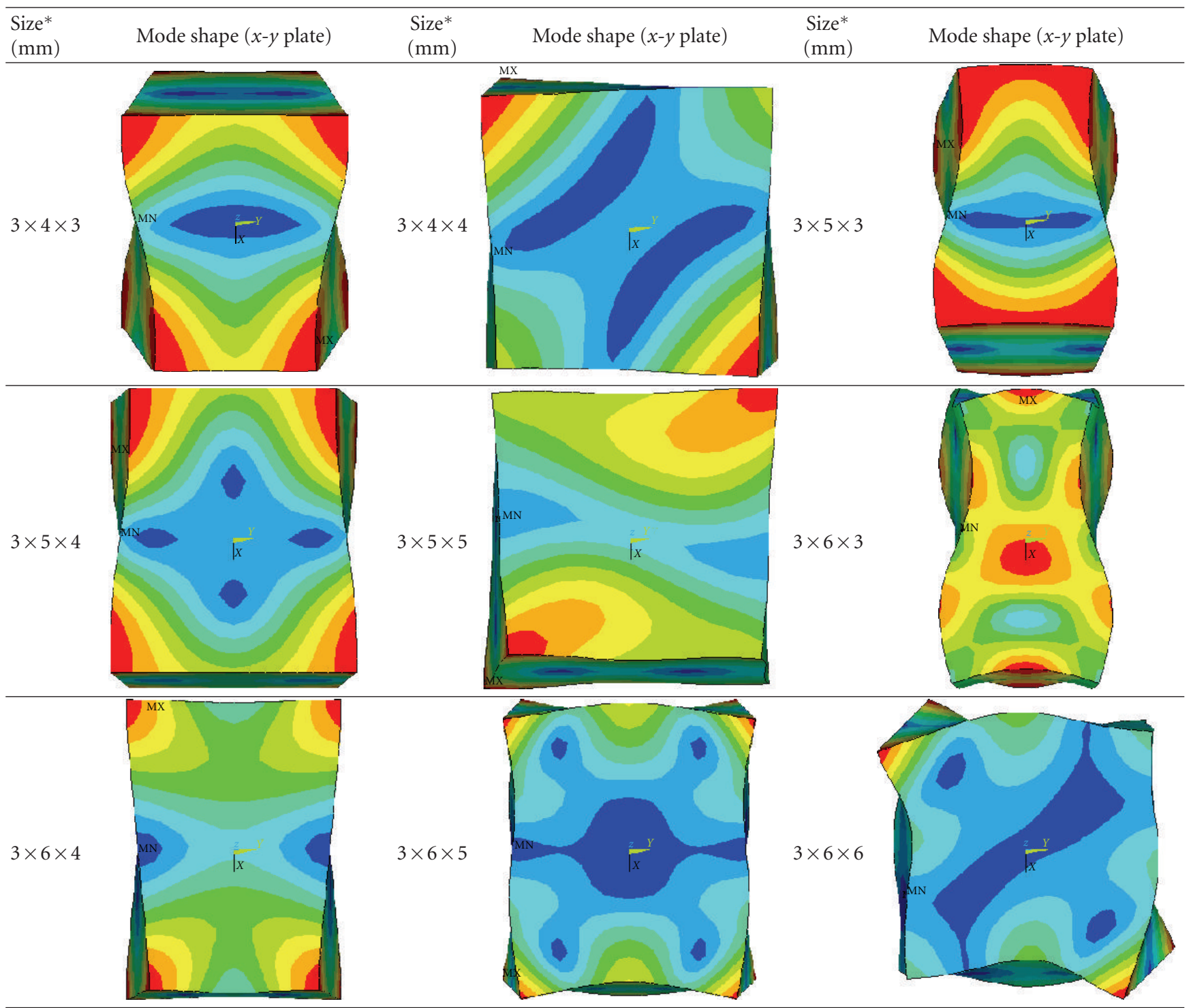

( ${ }^{*}$ The first number of the size refers to the size in the polarization direction).

TABLE 2: Calculating results of quantitative indicators.

\begin{tabular}{|c|c|c|c|c|c|c|}
\hline Size* & $\mathrm{F}(\mathrm{Hz})$ & $A_{x}$ & $A_{y}$ & $A_{z}$ & $A\left(E_{y} / E_{x}\right)$ & $B\left(E_{y} / E_{z}\right)$ \\
\hline $3 \times 4 \times 3$ & 388045 & $4.5464 e+006$ & $8.8189 e+005$ & $2.9196 e+006$ & 0.1940 & 0.3021 \\
\hline $3 \times 4 \times 4$ & 357044 & $2.1886 e+006$ & $4.0152 e+006$ & $2.1341 e+006$ & 1.8346 & 1.8814 \\
\hline $3 \times 5 \times 3$ & 348154 & $4.7358 e+006$ & $7.3201 e+005$ & $2.8966 e+006$ & 0.1546 & 0.2527 \\
\hline $3 \times 5 \times 4$ & 326550 & $2.6398 e+006$ & $3.0201 e+006$ & $2.7044 e+006$ & 1.1441 & 1.1167 \\
\hline $3 \times 5 \times 5$ & 372396 & $4.7605 e+006$ & $4.2082 e+005$ & $3.1742 e+006$ & 0.0884 & 0.1326 \\
\hline $3 \times 6 \times 3$ & 428269 & $4.7268 e+006$ & $1.4384 e+006$ & $2.1160 e+006$ & 0.3043 & 0.6798 \\
\hline $3 \times 6 \times 4$ & 370765 & $2.7430 e+006$ & $2.4641 e+006$ & $3.1312 e+006$ & 0.8983 & 0.7870 \\
\hline $3 \times 6 \times 5$ & 427822 & $3.9516 e+006$ & $2.8394 e+006$ & $1.4470 e+006$ & 0.7185 & 1.9623 \\
\hline $3 \times 6 \times 6$ & 389186 & $3.7984 e+006$ & $2.2963 e+006$ & $2.2245 e+006$ & 0.6045 & 1.0323 \\
\hline
\end{tabular}

( $*$ The first number of the size refers to the size in the polarization direction). 


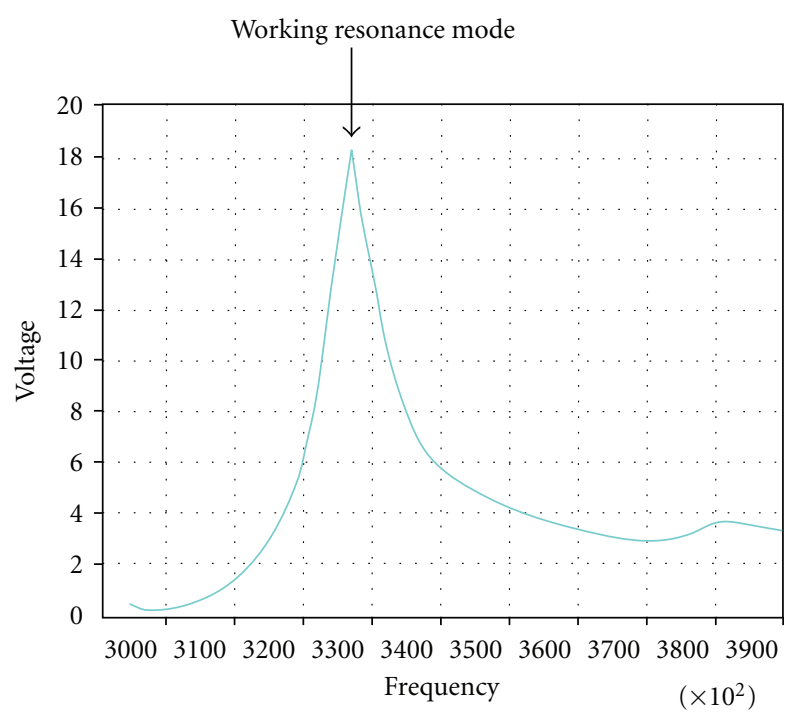

(a)

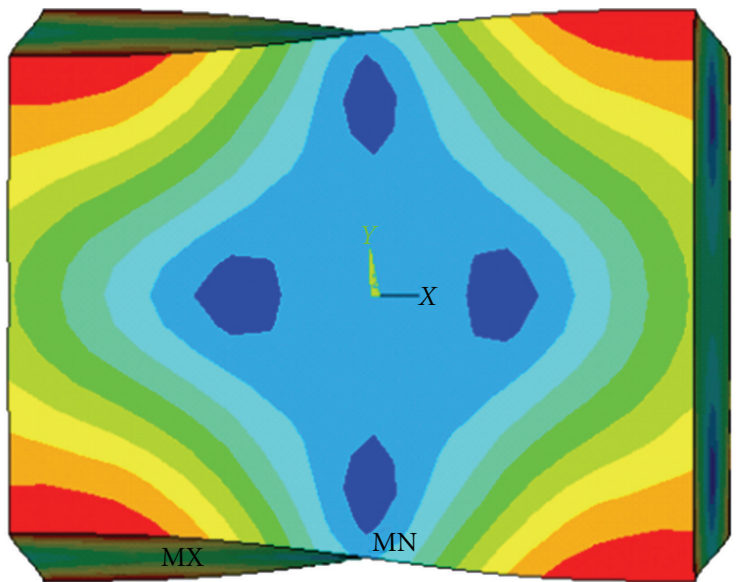

(b)

FIGURE 4: Harmonic excitation analysis result of the PMMG.

3.3. Harmonic Analysis. In real applications, we need to apply a driving voltage to actuate the device. To make sure the mode shape we activate is the same as the one we get in modal analysis, we used ANSYS to perform harmonic analysis. The configuration of the driving electrodes is shown in Figure 3. The amplitude of driving voltage is $10 \mathrm{~V}$, and the phase difference of the voltages on $\mathrm{D}+$ and $\mathrm{D}-$ is $180^{\circ}$. The damping constant of the piezoelectric material was introduced with the value of 0.02 . The frequency of the driving voltage was scanned from $300 \mathrm{kHz}$ to $500 \mathrm{kHz}$. Figure 4 gives the harmonic analysis results of the PMMG. In Figure 4(a), the $x$-coordinate refers to the frequency of the driving voltage and the $y$-coordinate refers to the piezoelectric voltage amplitude on the reference electrode, R1 or R2. From the figure, we can see that there are two peaks of the voltage of the reference electrode. The peak corresponding to the working resonance mode is in the position of around $325 \mathrm{kHz}$. The frequency of the working resonance mode is $326550 \mathrm{~Hz}$. Figure 4(b) shows the mode shape at the exciting frequency of the working resonance mode. It is concluded that the exciting vibration of the piezoelectric block is the same as the vibrating shape of the working resonance mode. That is to say that through applying driving voltage with the frequency of the working resonance mode on the driving electrodes, the vibration of the working resonance mode can be actuate.

Then, we need to optimize the size and configuration of the driving electrode to actuate the device more efficiently. Similar to modal analysis, we used $A$ and $B$ to serve as evaluation indicators. When $A$ and $B$ get bigger, that means the movements of the mass elements are more obvious at $Y$ direction than at $X$ and $Z$ directions, and we can say the device is actuated more efficiently. The original configuration of the driving electrodes is shown in

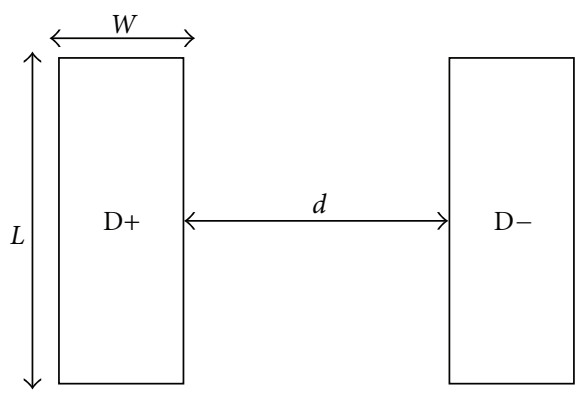

FIGURE 5: The original configuration and optimizing variables of the driving electrodes.

Figure 5. To optimize the size of the driving electrodes, we introduced three variables that can be changed: the length and width of the driving electrodes and the distance between two adjacent electrodes, which is shown schematically in Figure 5.

We changed one variable at a time and keet the other two variables unchanged; Table 3 shows the calculating results of quantitative indicators.

From Table 3 we can see that the maximum value of $A$ is around 0.68 , which means the movements of the mass elements are more obvious at $X$ direction than at $Y$ direction and may impact the performance of the device. So we need to increase the value of $A$ as well as $B$. To do this, we should find a way to diminish the movements of the mass elements in the $X$ direction. A simple solution is to add two additional driving electrodes (D3 and D4) distributed perpendicular to the previous electrodes (D1 and D2) as shown in Figure 6. The phase of voltage applied on electrode D3 is same as that of D1, and the phase of voltage applied on D4 is same as that 
TABLE 3: Relation between the quantitative indicators and the optimizing variables.

\begin{tabular}{|c|c|c|c|c|c|c|}
\hline Variable & Value $(\mathrm{mm})$ & $A_{x}$ & $A_{y}$ & $A_{Z}$ & $A\left(E_{Y} / E_{X}\right)$ & $B\left(E_{Y} / E_{Z}\right)$ \\
\hline \multirow{3}{*}{$d(W=0.75 \mathrm{~mm}, L=1 \mathrm{~mm})$} & 0.5 & $6.8325 e-015$ & $3.8273 e-015$ & $1.3724 e-014$ & 0.5602 & 0.2789 \\
\hline & 1 & $6.7979 e-015$ & $4.0901 e-015$ & $1.3562 e-014$ & 0.6017 & 0.3016 \\
\hline & 1.5 & $6.1609 e-015$ & $4.1988 e-015$ & $1.2567 e-014$ & 0.6815 & 0.3341 \\
\hline \multirow{4}{*}{$W(d=0.5 \mathrm{~mm}, L=1 \mathrm{~mm})$} & 0.45 & $3.1305 e-015$ & $1.9173 e-015$ & $6.4646 e-015$ & 0.6125 & 0.2966 \\
\hline & 0.75 & $6.8325 e-015$ & $3.8273 e-015$ & $1.3724 e-014$ & 0.5602 & 0.2789 \\
\hline & 1.05 & $7.3949 e-015$ & $4.4588 e-015$ & $1.4731 e-014$ & 0.6030 & 0.3027 \\
\hline & 1.35 & $7.3145 e-015$ & $5.0010 e-015$ & $1.4777 e-014$ & 0.6837 & 0.3384 \\
\hline \multirow{4}{*}{$L(d=0.5 \mathrm{~mm}, W=0.75 \mathrm{~mm})$} & 0.5 & $3.8474 e-015$ & $2.2331 e-015$ & $7.8047 e-015$ & 0.5804 & 0.2861 \\
\hline & 1 & $6.8325 e-015$ & $3.8273 e-015$ & $1.3724 e-014$ & 0.5602 & 0.2789 \\
\hline & 1.5 & $8.8826 e-015$ & $5.1518 e-015$ & $1.7828 e-014$ & 0.5800 & 0.2890 \\
\hline & 2 & $1.0169 e-014$ & $6.4411 e-015$ & $2.0583 e-014$ & 0.6334 & 0.3129 \\
\hline
\end{tabular}

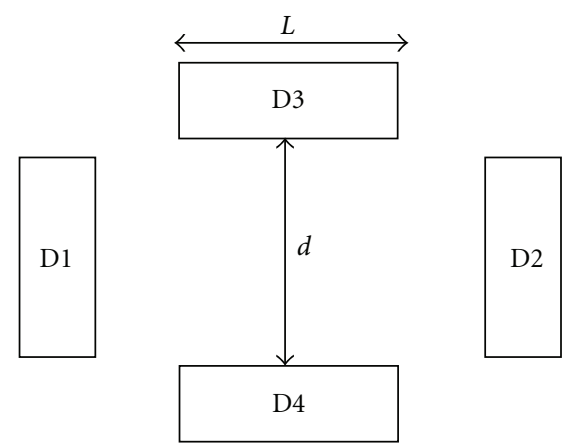

FIGURE 6: The new configuration and optimizing variables of the driving electrodes.

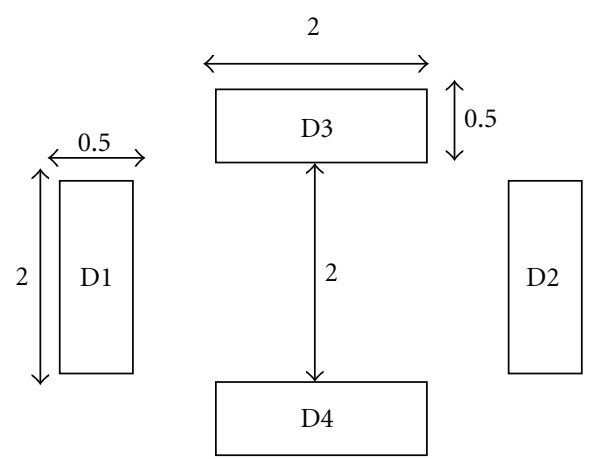

Figure 7: The configuration of the driving electrodes at Coriolis analysis.

of D2. To further optimize the size of the additional driving electrodes, we introduced three variables which should be changed: the length of the driving electrodes, the voltage applied on the driving electrodes, and the distance between two adjacent electrodes.

We changed one variable at a time and keep the other two variables unchanged; Table 4 shows the calculating results of quantitative indicators.

It can be concluded from Table 4 that the values of $A$ and $B$ increase to 0.73 and 0.62 , respectively, because of the application of these two additional driving electrodes.

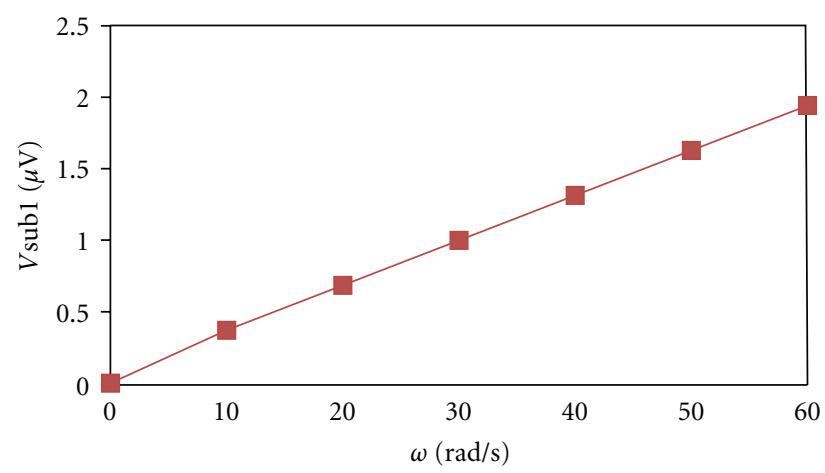

(a)

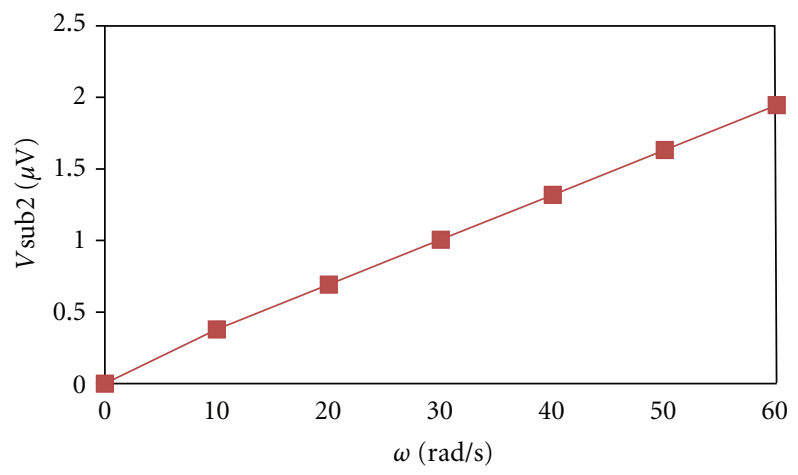

(b)

FIGURE 8: Relation between $V_{\text {sub }}$ and angular velocity applied to the device.

3.4. Coriolis Analysis. As mentioned before, when the angular velocity along $x$-axis is applied, the Coriolis force is generated according to movement of the mass elements, resulting in compressive and tensile stresses depending on the position. These stresses differentially generate the piezoelectric voltage at the surface of the device as shown in Figure 1(b). This voltage is proportional to the applied angular rate and is an output signal of the device.

With excitation at operation mode, we changed the angular velocity applied to the device, and surface potential at the detecting electrodes was calculated by ANSYS taking 
TABLE 4: Relation between the quantitative indicators and the optimizing variables.

\begin{tabular}{|c|c|c|c|c|c|c|}
\hline Variable & Value & $A_{x}$ & $A_{y}$ & $A_{Z}$ & $A\left(E_{Y} / E_{X}\right)$ & $B\left(E_{Y} / E_{Z}\right)$ \\
\hline \multirow{4}{*}{$L(\mathrm{~mm})(d=0.5 \mathrm{~mm}, V=1 \mathrm{~V})$} & 0.5 & $5.3504 e-015$ & $3.8128 e-015$ & $1.1329 e-014$ & 0.7126 & 0.3366 \\
\hline & 1 & $4.6164 E-15$ & $3.3119 E-15$ & $1.0090 E-14$ & 0.7174 & 0.3282 \\
\hline & 1.5 & $3.5850 E-15$ & $2.5867 E-15$ & $8.1780 E-15$ & 0.7215 & 0.3163 \\
\hline & 2 & $2.5532 E-15$ & $1.8392 E-15$ & $6.0732 E-15$ & 0.7204 & 0.3028 \\
\hline \multirow{4}{*}{$d(\mathrm{~mm})(L=2 \mathrm{~mm}, V=1 \mathrm{~V})$} & 0.4 & $4.09 E-15$ & $2.94 E-15$ & $8.9969 E-15$ & 0.7205 & 0.3272 \\
\hline & 1 & $3.87 E-15$ & $2.79 E-15$ & $8.6332 E-15$ & 0.7218 & 0.3232 \\
\hline & 1.6 & $3.5850 E-15$ & $2.5867 E-15$ & $8.1780 E-15$ & 0.7215 & 0.3163 \\
\hline & 2 & $3.47 E-15$ & $2.50 E-15$ & $7.9879 E-15$ & 0.7212 & 0.3130 \\
\hline \multirow{4}{*}{$V(V)(L=2 \mathrm{~mm}, D=0.5 \mathrm{~mm})$} & 0.5 & $3.4851 E-15$ & $2.5637 E-15$ & $4.0983 E-15$ & 0.7356 & 0.6255 \\
\hline & 1 & $2.5532 E-15$ & $1.8392 E-15$ & $6.0732 E-15$ & 0.7204 & 0.3028 \\
\hline & 1.5 & $3.7613 E-15$ & $2.6388 E-15$ & $8.4448 E-15$ & 0.7016 & 0.3125 \\
\hline & 2 & $2.4370 E-14$ & $8.5989 E-15$ & $4.3082 E-14$ & 0.3528 & 0.1996 \\
\hline
\end{tabular}

account of the Coriolis force. Because the raw result of the output voltage for the applied angular velocity is differential with respect to $x=0$, we applied subtraction of the potentials at symmetric points, that is, $V$ sub1 $=V_{\mathrm{D} 1}-V_{\mathrm{D} 2}$ and $V$ sub2 $=V_{\mathrm{D} 3}-V_{\mathrm{D} 4}$. Figure 7 shows the configuration of the driving electrodes, and Figures 8(a) and 8(b) show the relation between $V$ sub1, $V$ sub2 and angular velocity applied to the device, respectively.

As we can see from Figure 8, Vsub1 and Vsub2 have a liner relation with angular velocity, which agrees with the working principle of PMMG and testifies that the rotation input can be quantized through detecting the voltage difference of two adjacent sensing electrodes.

\section{Conclusions}

The structure of the PMMG has larger stiffness, so it is resistant to shake and strike. In this paper, modal analysis was firstly conducted to determine the best operation mode for PMMG, and we developed a set of quantitative indicators to evaluate various operation modes. It can be concluded from that the device with the size $3 \times 5 \times 4$ has the best operation mode. Then, harmonic analysis was conducted to verify that the vibration of the working resonance mode can be obtained through applying driving voltage with the frequency of the working resonance mode on the driving electrodes. We also used these quantitative indicators introduced in modal analysis to optimize the size and the configuration of the driving electrodes to actuate the device more efficiently. The calculation results of the quantitative indicators show that the original configuration of the driving electrodes has poor value of $A$ and $B$, and the value of $A$ and $B$ will increase considerably because of the application of two additional driving electrodes distributed perpendicular to original driving electrodes. At last, Coriolis analysis was conducted, which shows that the voltage difference of two adjacent sensing electrodes caused by Coriolis effect has a liner relation with angular velocity, and that agrees with the working principle of PMMG.

The work in this paper provides a theoretical basis for realizing this novel kind of micromachined gyroscope.

\section{Outlook}

The main purpose of this paper is to provide a method to optimize the size of the device as well as the way to efficiently actuate the operation mode we needed. At present, it is almost impossible for us to simulate the device with any size because it is too time-consuming. Our subsequent research will further improve the optimizing results and put more focus on the theoretical derivation of the optimizing process.

\section{Acknowledgments}

Financial supports from the National Natural Science Foundation of China (50805096/E051202) and Defense Key Laboratory Foundation (2009-2011) are gratefully acknowledged.

\section{References}

[1] K. M. Killian, M. Burmenko, and W. Hollinger, "Highperformance fiber optic gyroscope with noise reduction," in Proceedings of the 12th Fiber Optic and Laser Sensors (SPIE '94), vol. 2292, pp. 255-263, November 1994.

[2] M. O. Scully and J. P. Dowling, "Quantum-noise limits to matter-wave interferometry," Physical Review A, vol. 48, no. 4, pp. 3186-3190, 1993.

[3] T. L. Gustavson, A. Landragin, and M. A. Kasevich, "Rotation sensing with a dual atom-interferometer Sagnac gyroscope," Classical and Quantum Gravity, vol. 17, no. 12, pp. 2385-2398, 2000.

[4] A. Matthews and F. J. Rybak, "Comparison of hemispherical resonator gyro and optical gyros," IEEE Aerospace and Electronic Systems Magazine, vol. 7, no. 5, pp. 40-46, 1992.

[5] M. Kurosawa, Y. Fukuda, M. Takasaki, and T. Higuchi, "A surface-acoustic-wave gyro sensor," Sensors and Actuators A, vol. 66, no. 1-3, pp. 33-39, 1998.

[6] S. W. Lee, J. W. Rhim, S. W. Park, and S. S. Yang, "A micro rate gyroscope based on the SAW gyroscopic effect," Journal of Micromechanics and Microengineering, vol. 33, pp. 1975-1977, 2007.

[7] P. Mottier and P. Pouteau, "Solid state optical gyrometer integrated on silicon," Electronics Letters, vol. 33, no. 23, pp. 1975-1977, 1997. 
[8] W. Guo, H. Ma, Z. Jin, Y. Z. Tang, and Y. L. Wang, "A novel structure of passive ring waveguide resonator on silicon substrate," in Proceedings of the SPIE, vol. 4928, pp. 297-300, October 2002.

[9] J. N. Sharma, V. Walia, and S. K. Gupta, "Effect of rotation and thermal relaxation on Rayleigh waves in piezothermoelastic half space," International Journal of Mechanical Sciences, vol. 50, no. 3, pp. 433-444, 2008.

[10] J. Soderkvist, "Piezoelectric beams and vibrating angular rate sensors," IEEE Transactions on Ultrasonics, Ferroelectrics, and Frequency Control, vol. 38, no. 3, pp. 271-280, 1991.

[11] C. S. Chou, J. W. Yang, Y. C. Hwang, and H. J. Yang, "Analysis on vibrating piezoelectric beam gyroscope," International Journal of Applied Electromagnetics in Materials, vol. 2, no. 3, pp. 227-241, 1991.

[12] I. A. Ulitko, "Mathematical theory of the fork-type wave gyroscope," in Proceedings of the 49th Annual IEEE International Frequency Control Symposium, pp. 786-793, June 1995.

[13] S. Fujishima, T. Nakamura, and K. Fujimoto, "Piezoelectric vibratory gyroscope using flexural vibration of a triangular bar," in Proceedings of the 45th Annual Symposium on Frequency Control, pp. 261-265, May 1991.

[14] G. M. Reese, E. L. Marek, and D. W. Lobitz, "Threedimensional finite element calculations of an experimental quartz rotation sensor," in Proceedings of the IEEE Ultrasonics Symposium, pp. 419-422, October 1989.

[15] H. Abe, T. Yoshida, T. Ishikawa, N. Miyazaki, and H. Watanabe, "Trapped-energy vibratory gyroscopes using a partially polarized piezoelectric ceramic plate," Electronics and Communications in Japan, vol. 284, pp. 798-805, 2001.

[16] J. S. Yang, H. Y. Fang, and Q. Jiang, "A vibrating piezoelectric ceramic shell as a rotation sensor," Smart Materials and Structures, vol. 9, no. 4, pp. 445-451, 2000.

[17] J. S. Burdess and T. Wren, "Theory of a piezoelectric disc gyroscope," IEEE Transactions on Aerospace and Electronic Systems, vol. 22, no. 4, pp. 410-418, 1986.

[18] J. S. Burdess, "The dynamics of a thin piezoelectric cylinder gyroscope," Proceedings of the Institution of Mechanical Engineers, vol. 200, pp. 271-280, 1986.

[19] P. W. Loveday, "A coupled electromechanical model of an imperfect piezoelectric vibrating cylinder gyroscope," Journal of Intelligent Material Systems and Structures, vol. 7, no. 1, pp. 44-53, 1996.

[20] K. Maenaka, H. Kohara, M. Nishimura, T. Fujita, and Y. Takayama, "Novel solid micro-gyroscope," in Proceedings of the 19th IEEE International Conference on Micro Electro Mechanical Systems, pp. 634-637, Istanbul, Turkey, January 2006.

[21] X. Wu, W. Chen, Y. Lu et al., "Vibratin analysis of a piezoelectric micromachined modal gyroscope," Journal of Micromechanics and Microengineering, vol. 19, no. 12, Article ID 125008, 2009.

[22] W. K. Sung, M. Dalal, and F. Ayazi, "mode-matched $0.9 \mathrm{MHz}$ single proof-mass dual-axis gyroscope," in Proceedings of the 16th International Solid-State Sensors, Actuators and Microsystems (TRANSDUCERS '11), pp. 2821-2824, Beijing, China, June 2011.

[23] B. J. Gallacher, J. S. Burdess, A. J. Harris, and M. E. McNie, "Principles of a three-axis vibrating gyroscope," IEEE Transactions on Aerospace and Electronic Systems, vol. 37, no. 4, pp. 1333-1343, 2001. 

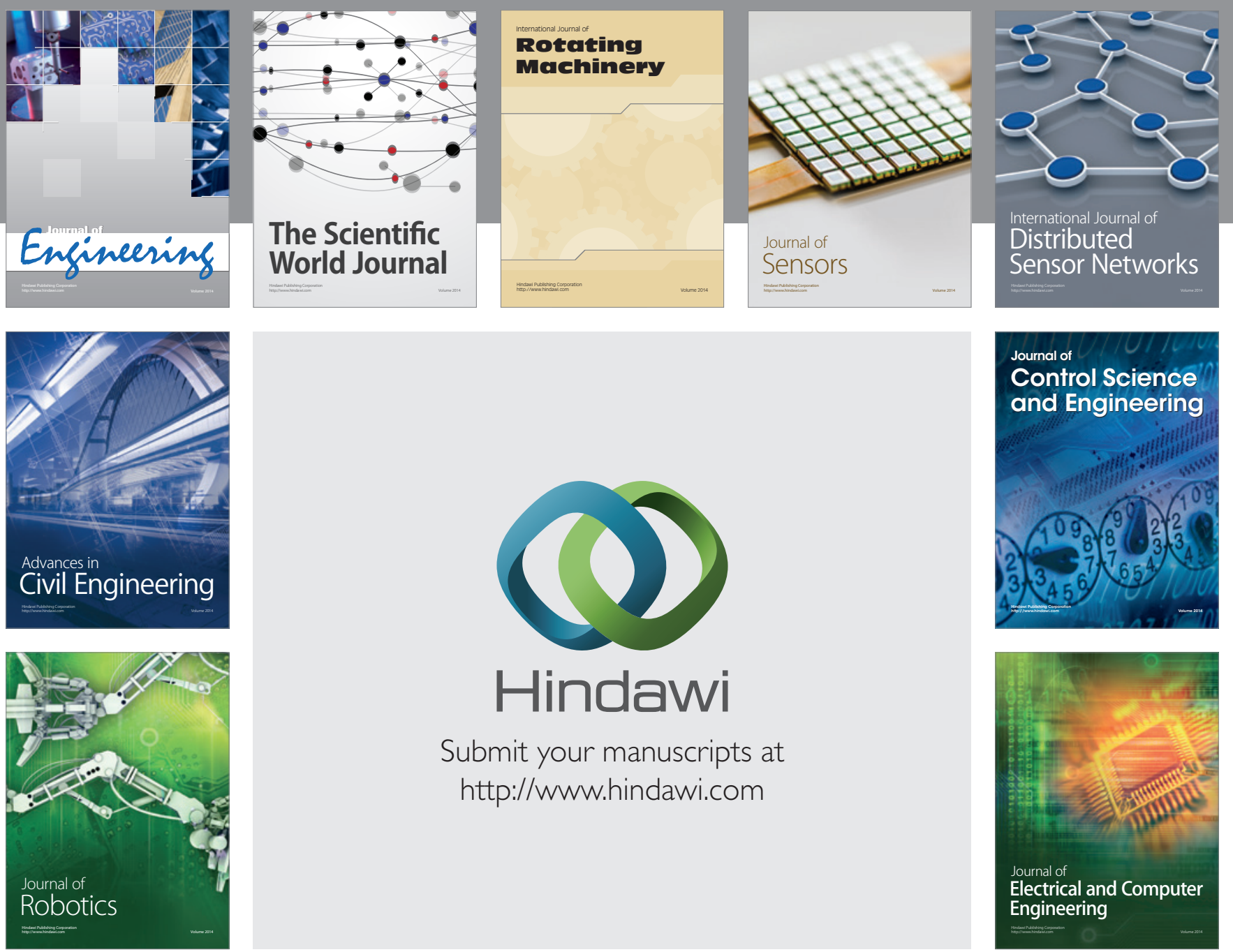

Submit your manuscripts at

http://www.hindawi.com
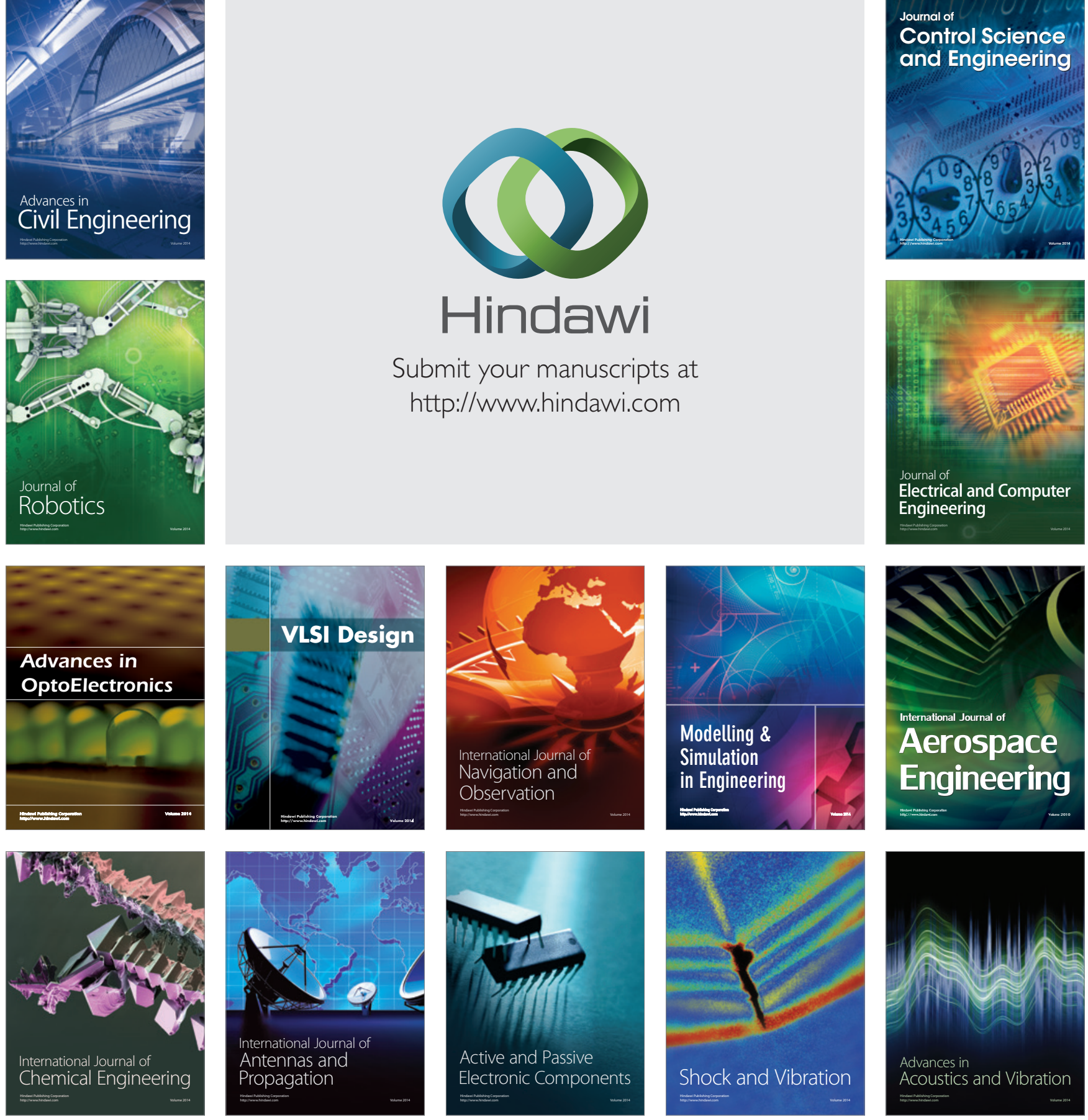